\title{
BMJ Open Urban scaling of health outcomes: a protocol for a scoping review
}

\author{
Edwin M McCulley (D) , ${ }^{1,2}$ Pricila Mullachery, ${ }^{1}$ Daniel Rodriguez, ${ }^{3,4}$ \\ Ana V Diez Roux, ${ }^{1,2}$ Usama Bilal (i) ${ }^{1,2}$
}

To cite: McCulley EM, Mullachery P, Rodriguez D, et al. Urban scaling of health outcomes: a protocol for a scoping review. BMJ Open 2019;9:e031176. doi:10.1136/ bmjopen-2019-031176

- Prepublication history and additional material for this paper are available online. To view these files, please visit the journal online (http://dx.doi. org/10.1136/bmjopen-2019031176).

Received 19 April 2019 Revised 06 November 2019 Accepted 07 November 2019

Check for updates

(C) Author(s) (or their employer(s)) 2019. Re-use permitted under CC BY-NC. No commercial re-use. See rights and permissions. Published by BMJ.

${ }^{1}$ Urban Health Collaborative, Drexel University Dornsife School of Public Health, Philadelphia, Pennsylvania, USA ${ }^{2}$ Department of Epidemiology and Biostatistics, Drexel University Dornsife School of Public Health, Philadelphia, Pennsylvania, USA

${ }^{3}$ Department of City and Regional Planning, University of California Berkeley, Berkeley, California, USA

${ }^{4}$ Institute for Transportation Studies, University of California Berkeley, Berkeley, California, USA

Correspondence to

Dr Usama Bilal;

ubilal@drexel.edu

\begin{abstract}
Introduction With most of the world's population living in urban areas, it is important to understand the health effects of city living. Precise descriptions of the relations of city size and growth with population health metrics have not been systematically described. Describing these relationships can provide clues regarding the factors driving differences in health across cities.

Objective The goal of this scoping review is to map the existing evidence regarding the scaling properties of health outcomes, with a special emphasis on city size and
\end{abstract} growth.

Method and analysis We will conduct this scoping review based on a structured search of a combination of keywords for the exposures that aim to capture the population growth and size of cities along with degree of urbanisation, for health-related outcomes regardless of their type (incidence or prevalence of diseases and injuries and mortality). We will search the MEDLINE and Latin American \& Caribbean Health Sciences Literature databases from inception for studies in English, Spanish or Portuguese and perform manual searches of references and citations in the included studies. The search will be conducted from April 2019 to September 2019. We will report results using the PRISMA-ScR guidelines. This review aims to inform future research on the role of increasing levels of urbanisation on health outcomes, provide clues into what city level factors may drive patterns of urban health and lead to the design and implementation of policies that promote sustainable growth and improved health.

Ethics and dissemination This review will not collect any primary data and will rely on published articles. The findings of this review will be published in a peer-reviewed scientific journal.

\section{INTRODUCTION}

High rates of urbanisation worldwide warrant attention on the drivers of health in cities. ${ }^{1}$ The New Urban Agenda and the Sustainable Development Goals increasingly recognise the importance of improving urban health in achieving other development goals. ${ }^{2} 3$ There are insufficient data on how cities vary in health profiles or how variations in these profiles may be related to city level factors amenable to policy.

The emerging literature on urban scaling has shown how understanding the ways that
Strengths and limitations of this study

Mapping the scaling properties of cities on health outcomes can offer insights into the links between growing urbanisation and health.

- These associations are potentially heterogeneous and dependent on the definition of city size and growth and the outcome under study.

- Limiting eligible studies to those published in English, Spanish and Portuguese may exclude studies in other highly urbanised areas.

urban factors change with city size can help create a theory of city living. ${ }^{4-7}$ The idea of studying scaling traces back to the study of metabolic outcomes in mammals that has shown how some features such as the basal metabolic rate grow less than expected with animal size. ${ }^{4}$ This empirical finding led to the realisation that fractal structures in living systems (such as the capillary or pulmonary systems) optimise energy delivery and thus reduce the required rate of energy expenditure per unit of size. ${ }^{4}$ Applying the same framework to cities has led to analogous insights regarding the functioning of urban systems. ${ }^{5-7}$ Previous research has shown that some city features, such as energy expenditures, residue production (trash, pollution) and other outputs (wealth, crime $)^{6}$ change with city size. These changes describe the scaling properties of various city features, each of which has a unique relationship to city growth and size.

The processes (and scaling properties) linking urban size to health have not been thoroughly investigated. Some health outcomes have been examined in terms of their scaling properties, ${ }^{9}{ }^{10}$ but these have not been systematically summarised. All of these factors are potential determinants of health, and therefore cities of different sizes may experience different population health outcomes. However, to our knowledge, very little research has tried to understand these 
scaling properties and their relationship with population health metrics.

While size is usually considered as a static feature of cities, the current population of a city is the result of the dynamics of urban environments that grow at different rates and in different ways. ${ }^{5-7}$ Some cities have slow controlled growth, while others grow more rapidly, and others shrink over time. ${ }^{11}$ Some grow through sprawl, and others grow through increased density. The scaling properties of health outcomes may also be different when considering growth instead of size, given these different ways in which cities grow. ${ }^{12}$ Understanding the scaling properties of cities on health outcomes can help inform urban policy levers such as urban planning or health policies that may help improve population health under growing urbanisation levels.

\section{Objectives}

The goal of this scoping review is to map the existing evidence regarding the scaling properties of cities on health outcomes. This includes exploring the association of city size and growth with health outcomes, with the goal of understanding the effects of increasing urbanisation rates and potential policy levers that may modify this association. A clearer understanding of these links will allow public health practitioners and urban planners in understanding the effects of increasing rates of urbanisation and the potential benefits of policies that lead to sustainable growth.

There is no universally accepted definition of both city size and growth. Given our interest in examining the health effects of urban scaling, we define city size as the population (number of people) of a city's metropolitan or administrative area and city growth as the changes in population over time in a city's metropolitan or administrative area. In the review, we will examine population size in whole urban areas regardless of geographical unit (administrative, functional, built-up). Therefore, we will not be examining size or growth in terms of urban footprint, extent and sprawl.

\section{METHODS AND ANALYSIS \\ Design}

Preliminary searches for the scoping review described in this protocol began in April 2019 and ended in September 2019. The scoping review was conducted as part of the SALURBAL (Salud Urbana en America Latina) project. ${ }^{11}{ }^{11}$ Scoping reviews are used to examine existing literature pertaining to our study objectives: to map the available evidence pertaining to city size, city growth and health outcomes in terms of available research results, design and methodology. ${ }^{13}$ The scoping review framework was initially developed by Arksey and $\mathrm{O}^{\prime}$ Malley ${ }^{14}$ and has been further augmented by the work of Levac et al. ${ }^{15}$ These fundamental frameworks are both synthesised and drawn on in the Joanna Briggs Institute (JBI) methodology for conducting scoping reviews. ${ }^{16}$ In this study, the scoping review will be conducted following the methodology described by JBI. Existing literature addressing the scaling properties of health outcomes with city population size has yet to be comprehensively reviewed, as distinct studies relevant to our objectives continue to emerge. Peters $e t a l^{16}$ describe the benefits of this design, in that scoping reviews allow for the summary and dissemination of research findings, identify gaps in existing literature and can make recommendations relevant to the directions of future research. ${ }^{14}$ The research and writing group will follow the framework outlined in the Methodology for JBI Scoping Reviews ${ }^{16}$ and the 2018 guidelines for reporting described in Preferred Reporting Items for Systematic Review and Meta-Analysis Extension for Scoping Reviews (PRISMA-ScR). ${ }^{17}$ This scoping review protocol is ineligible for registration in the International Prospective Register of Systematic Reviews (PROSPERO). ${ }^{18}$

\section{Data selection}

\section{Search for electronic databases}

We will search two electronic databases: MEDLINE (via PubMed) and the Latin American \& Caribbean Health Sciences Literature (LILACS) database which incorporates literature pertaining to the various disciplines of health science in Spanish and Portuguese. These databases will be searched from inception, and the search will not be constrained to any specific time period. The Americas are one of the most urbanised regions in the world; therefore, limiting our search to only include publications in English may exclude relevant articles. In an attempt to maximise eligible studies and in keeping with the language expertise of the research team, the search will be executed in English, Spanish and Portuguese. This scoping review will be conducted following a structured search strategy based on key terms and ordered vocabulary relevant to our study objectives. Key terms affiliated with the inclusion criteria will include city size, urban size, city growth, urban growth, city scaling, urban scaling, urbanisation, urbanisation, incidence, prevalence, morbidity, mortality, health and disease. The search terms and parameters employed for this structured search are described in table 1 . The terms were translated into Spanish and Portuguese (see online supplementary appendix tables 1 and 2). Prior to executing the full search strategy outlined in the online supplementary appendix, a preliminary partial analysis on the number of studies expected of the scoping review was conducted using Google Scholar. This analysis used the search strategy outlined in table 1 and yielded 981 potentially eligible studies.

\section{Search for other resources}

In addition to examining the databases described above (MEDLINE via PubMed and LILACS), we will conduct both backward searches (screening references cited in included studies) and forward searches (exploring studies that cite included studies using Google Scholar). 
Table 1 Structured search strategy, key terms and search parameters

\begin{tabular}{ll}
\hline $\begin{array}{l}\text { Key terms related to } \\
\text { exposures }\end{array}$ & $\begin{array}{l}\text { Key terms related } \\
\text { to outcomes }\end{array}$ \\
\hline $\begin{array}{l}\text { 1. ('City Size' OR 'Urban } \\
\text { Size') }\end{array}$ & $\begin{array}{l}\text { (Incidence } \\
\text { OR } \\
\text { Prevalence }\end{array}$ \\
$\begin{array}{l}\text { 2. ('City Growth' OR } \\
\text { 'Urban Growth') }\end{array}$ & $\begin{array}{l}\text { OR } \\
\text { Morbidity } \\
\text { OR }\end{array}$ \\
$\begin{array}{l}\text { 3. ('City Scaling' OR } \\
\text { 'Urban Scaling') }\end{array}$ & $\begin{array}{l}\text { Mortality } \\
\text { OR } \\
\text { Health }\end{array}$ \\
4. ('Urbanization' OR & OR \\
'Urbanisation') & Disease) \\
\hline
\end{tabular}

Search parameters refer to the Boolean operators applied to the search terms used in the search strategy.

\section{Data analysis}

\section{Eligibility criteria}

We will include all research studies identified in the search with the objective to study any association between city size, city growth and health outcomes, regardless of study design (case reports, cross-sectional studies, case-control studies, cohort studies, randomised controlled trials, systematic reviews and meta-analyses). Given the objective of scoping reviews and the heterogeneous nature of both the exposure and outcome variables, eligible studies will not be evaluated for inclusion based on the quality of the research (bias, confounders and so on). Furthermore, studies eligible for inclusion will not be evaluated on the type of research employed (qualitative or quantitative), nor the type of urbanisation process (planned or unplanned). All study populations, regardless of sociodemographic factors (age, gender, migration status and so on) are eligible for inclusion. For the reasons outlined above, our search strategy is limited to studies published in English, Spanish and Portuguese. Additionally, literature eligible for inclusion is not restricted by any date or study period constraints.

\section{Exclusion criteria}

Our scoping review aims to map evidence related to our objectives; thus, opinion-based publications such as letters, commentaries and editorial reviews will be excluded. Studies with primary objectives other than those analysing potential associations between city size, city growth and health outcomes will be excluded from the scoping review. Moreover, studies written in languages other than English, Spanish and Portuguese will be excluded, due to the expertise of the research team.

\section{Data extraction items}

Conducive to exploring the scaling properties of health outcomes with city population size, we will extract the following data from eligible literature identified in our search:
- Study attributes: author(s), publication date, title, journal and citation.

- Study objectives.

- Study design: longitudinal or cross-sectional, ecological or individual-level, qualitative or quantitative.

- Study population: population of interest, population setting and settlement classification.

- City definition: official country-defined metropolitan areas, administrative areas (eg, counties), commuting zones, urban extent and so on.

- Exposure measures: size measure used to quantify population growth, definition of population size and/ or population growth.

- Outcome measures: measures used to quantify health outcomes relative to search terms.

- Study results and relevant conclusion(s), effect on health outcomes and directions for future research.

- Any additional relationships or associations relevant to scoping review objectives that may be of interest to the research team.

\section{Data management}

References identified through the electronic database searches will be downloaded and compiled with literature identified using other resources. These references will be compiled into an EndNote library and will be screened for duplicates. References will then be imported into Covidence for evidence synthesis and screening. All references will be screened by two reviewers using a three-step approach, which includes title, abstract and full-text evaluation. Using Covidence, a preliminary data extraction form will be used and tested on 10 studies. This form will be modified by reviewers as needed in order to compare and quantify the evidence described in the studies. Results from the screening and selection process of the search will be clearly documented using a PRISMA flow diagram. Potential disagreements arising from the screening and selection process will be recorded, discussed and resolved by reviewers.

\section{Data synthesis}

Statistical methods of synthesis such as meta-analysis will not be performed in this review; this is largely due to the heterogeneous nature of the evidence. Scoping reviews aim to examine broad areas of research, identify gaps in existing literature and map relevant evidence. Characteristics related to city size, city growth and health outcomes will be qualitatively synthesised and summarised in the Results section. Quantitative data will undergo summary analysis and will be reported in tables and figures when necessary. Publications and search decisions will be summarised using the PRISMA Flow Chart Diagram for the scoping review process. ${ }^{18}$ The researchers will map the body of evidence from all included literature, examine the evidence in terms of the review objectives and provide direction for future research on the topic. To provide a narrative summary of data synthesis, the PRISMA-ScR statement for reporting will be used in 
presenting the methods and findings of the scoping review. ${ }^{17}$

\section{Patient and public involvement}

We conducted a Knowledge to Policy Forum in November of 2018 in Mexico City to assess priorities in urban health by policy actors in the Latin American region. For more information, check https://drexel.edu/lac/events-workshops/knowledge-policy-forum/

\section{CONCLUSION}

The scoping review will map existing literature pertaining to the scaling properties of cities on health outcomes relative to urbanisation levels, city size and population growth. The findings of the scoping review will provide an overview of the body of literature examining the scaling properties of health outcomes with city population size and urban growth. A clearer understanding of the role of city size and growth on determining health outcomes will help further understand the effects of increasing urbanisation rates, allowing public health practitioners and urban planners in the design of policies that lead to sustainable growth.

Contributors UB and EMM conceived the study and drafted the first version of the protocol. UB obtained funding for the study. PM, DR and AVDR contributed to the revision and improvement of the protocol design. All authors critically reviewed the protocol for important intellectual content and gave final approval of the protocol version to be submitted for publication. UB, EMM, PM, DR and AVDR are accountable for all aspects of the protocol and will ensure that questions related to the integrity or accuracy of the protocol are properly investigated and resolved accordingly.

Funding This work was supported by the Office of the Director of the National Institutes of Health under award number DP50D26429. AVDR and DR were also supported by the Welcome Trust initiative, 'Our Planet, Our Health' (Grant 205177/Z/16/Z).

Competing interests None declared.

Patient consent for publication Not required.

Provenance and peer review Not commissioned; externally peer reviewed.

Open access This is an open access article distributed in accordance with the Creative Commons Attribution Non Commercial (CC BY-NC 4.0) license, which permits others to distribute, remix, adapt, build upon this work non-commercially, and license their derivative works on different terms, provided the original work is properly cited, appropriate credit is given, any changes made indicated, and the use is non-commercial. See: http://creativecommons.org/licenses/by-nc/4.0/.

ORCID iDs

Edwin M McCulley http://orcid.org/0000-0003-3784-9991

Usama Bilal http://orcid.org/0000-0002-9868-7773

\section{REFERENCES}

1 UN-HABITAT. Urbanization and development: emerging futures; world cities report 2016. In: World cities report. Nairobi: UN-HABITAT, 2016.

2 Satterthwaite D. A new urban agenda? London, England: SAGE Publications, 2016: 28. 3-12.

3 Diez Roux AV, Slesinski SC, Alazraqui M, et al. A novel international partnership for actionable evidence on urban health in Latin America: LAC-Urban health and SALURBAL. Glob Chall 2019;3.

4 West G. Scale: Orion publishing group, limited, 2017.

5 Bettencourt L, West G. A unified theory of urban living. Nature 2010;467:912-3.

6 Bettencourt LMA. The origins of scaling in cities. Science 2013;340:1438-41.

7 Bettencourt LMA, Lobo J, Helbing D, et al. Growth, innovation, scaling, and the pace of life in cities. Proc Natl Acad Sci U S A 2007;104:7301-6.

8 West GB, Brown JH, Enquist BJ. A general model for the origin of allometric scaling laws in biology. Science 1997;276:122-6.

9 Patterson-Lomba O, Goldstein E, Gómez-Liévano A, et al. Per capita incidence of sexually transmitted infections increases systematically with urban population size: a cross-sectional study. Sex Transm Infect 2015;91:610-4.

10 Rocha LEC, Thorson AE, Lambiotte R. The non-linear health consequences of living in larger cities. Journal of Urban Health 2015;92:785-99.

11 Pumain D, Paulus F, Vacchiani-Marcuzzo C, et al. An evolutionary theory for interpreting urban scaling laws. Cybergeo 2006.

12 Depersin J, Barthelemy M. From global scaling to the dynamics of individual cities. Proc Natl Acad Sci U S A 2018;115:2317-22.

13 Peters MDJ. In no uncertain terms: the importance of a defined objective in scoping reviews. JBI Database System Rev Implement Rep 2016;14:1-4.

14 O'Malley L. Scoping studies: towards a methodological framework AU - Arksey, Hilary. Int J Soc Res Methodol 2005;8:19-32.

15 Levac D, Colquhoun H, O'Brien KK. Scoping studies: advancing the methodology. Implement Sci 2010;5.

16 Peters MDJ, Godfrey CM, Khalil H, et al. Guidance for conducting systematic scoping reviews. Int J Evid Based Healthc 2015;13:141-6.

17 Tricco AC, Lillie E, Zarin W, et al. PRISMA extension for scoping reviews (PRISMA-ScR): checklist and explanation. Ann Intern Med 2018;169:467-73.

18 Moher D, Shamseer L, Clarke M, et al. Preferred reporting items for systematic review and meta-analysis protocols (PRISMA-P) 2015 statement. Syst Rev 2015;4:1. 